\title{
The current therapeutic strategies of COVID-19
}

\author{
Zhichao Chen ${ }^{1, \mathrm{a},{ }^{*}, \dagger}$, Wenxi Miao ${ }^{2, \mathrm{~b},{ }^{*}, \dagger}$, Leran $\mathrm{Xu}^{3, \mathrm{cc},{ }^{*},}$, Qi Zhang ${ }^{4, \mathrm{~d},{ }^{*}, \dagger}$ \\ ${ }^{1}$ School of public health, Xiamen University, 361005, Xiamen, China \\ ${ }^{2}$ Suzhou Science \& Technology Town Foreign Language School, 215010, Suzhou, China \\ ${ }^{3}$ Nanjing Foreign Language School - British Columbia Academy, 210024, Nanjing, China \\ ${ }^{4}$ Suzhou Dulwich International High School, 215000, Suzhou, China \\ $†$ These authors contributed equally.
}

\begin{abstract}
At the beginning of 2020, the Covid-19 epidemic broke out and soon became a global public health problem. Therefore, many countries are speeding up the development of antiviral drugs and COVID-19 vaccines to achieve effective prevention, control, and treatment of Covid-19. This paper reviews the pathogenic mechanism and detection and diagnosis of COVID-19 and the status and research progress of antiviral drugs, vaccines, and some other treatments against it based on previous articles published in this field. Although Covid-19's pathogenic mechanism has been generally figured out and the detection methods are advanced, old pneumonia treatment methods are adopted Covid-19 due to the lack of high-efficiency antiviral drugs. Meanwhile, as more and more safe and effective vaccines enter the clinical phase, the treatment of the Covid-19 epidemic may rely more on the prevention-oriented strategy.
\end{abstract}

\section{Introduction}

According to the World Health Organization (WHO), Coronavirus disease (COVID-19) is an infectious disease caused by a newly discovered coronavirus [1]. Due to its high infectivity and high concealment, quarantine and isolation were the best protection measure for a long time. And different countries established different methods due to their cultures and situations to prevent the COVID-19. Though there are many drugs for COVID-19, the specific remedy has not been developed for a long time. Vaccines have become the focus of social attention. Moreover, it bonded with residents and gave people a deeper feeling of a community with a shared future for mankind. In this article, we first provide brief information about the COVID-19 and the current issue of prevention, then discuss the treatments to offer a clear understanding of the up-to-date situation during the pandemic period.

\section{Covid-19}

Severe acute respiratory syndrome coronavirus 2 (SARSCoV-2), the World Health Organization named coronavirus disease (COVID-19), is one of the highly pathogenic beta-coronaviruses infects humans. It is first emerged in Wuhan, China, on 8 December 2019, with symptoms of fever, cough, and muscle ache, often with progressive difficulty in breathing [1].

COVID-19 is a positive-sense single-stranded RNA virus. The length of the genome is approximately 26-32 $\mathrm{bp}$, which is the longest among all the RNA viruses. The

\footnotetext{
${ }^{\text {}}$ Corresponding author: ${ }^{\mathrm{a}} \mathrm{zcchen} @$ stu.xmu.edu.cn,

bwency12003@outlook.com, ${ }^{\mathrm{c} x}$ xlr2004@163.com,

d zabrina.zhang23@dchigh-suzhou.cn.
}

envelope of the coronavirus is the phosphate bilayer, which is the same as cell membranes. On the envelope, there're four proteins coded by viral genomes, including $\mathrm{S}$ protein (spike glycoprotein), $\mathrm{M}$ protein (membrane protein), E protein (envelope protein), and HE protein (hemagglutinin-esterase).

The most significant protein in the pathogenic mechanism is $\mathrm{S}$ protein, which attaches the virus to its cellular receptor, angiotensin-converting enzyme 2 (ACE2). A defined receptor-binding domain (RBD) on $\mathrm{S}$ mediates this interaction [2]. ACE2 plays a vital role in normal physiological mechanisms, so after combining with coronavirus, it loses its functions and causes severe disorders in angiography, kidney, and lung [3].The COVID-19 mainly relies on the hydrolysis of proteins by the endosome or lysosome to enter the cells. When $\mathrm{S}$ protein reacts with ACE2 protein on the cellular surface, $\mathrm{S}$ protein can be divided into two subdomains, S1 and S2. The fusion peptide domain is attached to the host's cell membranes to shorten the virus and host cell's distance. After entering the host's cell, the virus release nucleocapsid (N protein and RNA) into the cytoplasm to translate and replicate. After translating, the viral protein can form a new virion and be released outside the cell by exocytosis.

\section{Early diagnosis of COVID-19}

The diagnostic tools are generally molecular methods, serology, and viral culture [3]. There're different levels of symptoms: for most common symptoms, such as fever, dry cough, and tiredness. In a few cases, patients may also 
get aches and pains, sore throat, diarrhoea, conjunctivitis, headache, loss of taste or smell, etc. The most serious symptoms are difficulty breathing or shortness of breath, chest pain or pressure, loss of speech or movement. One of the ways of diagnosing COVID-19 [4] is detecting viral genetic material (RNA) and detecting antibodies produced by our body in response to viral infection. The viral RNA assay is designed to detect the presence of SARS-CoV-2 RNA from a specimen collected from a nasal or throat swab of an infected person. On the other hand, antibody tests use an immunoassay to detect antibodies that may be used as an indicator of current or previous infection and are therefore complementary to viral RNA tests. More significantly, antibody testing provides information about the potential presence, duration, and intensity of acquired immunity. As shown in figure 4, there are four main types of immunoglobulin in primates, $\operatorname{IgG}, \operatorname{IgM}, \operatorname{IgA}$, and $\operatorname{IgE}$ [5]. IgM antibody is produced in about 5-7 days after infected by COVID-19, with short duration and fast disappearance. A positive blood test can judge early infection, which is conducive to early detection and early treatment. The IgG antibody is produced in 10-15 days and can remain in the blood circulation for a long time. The positive blood test can be used as an indicator of infection or previous infection. The antibody detection is faster, more convenient, and requires relatively low environmental conditions than the other methods to determine the coronavirus infection.

As the standard gold test for SARS-CoV-2 identification, real-time quantification RT-PCR (qRTPCR), with high sensitivity, is the routine confirmation test recommended by WHO. Samples from throats and nasal cavities are usually detected. However, due to the timeconsuming process, such as the high level of requirements of expensive equipment and biosafety conditions, this test is not suitable for point-of-care diagnosis. In addition, point-of-care tests are suitable for clinical laboratories. They can be performed by trained non-laboratory personnel in patient care facilities, such as physicians' offices or Emergency Departments, making the diagnostic test of SARS-CoV-2 closer to the patient.

Both of the two methods separately play an important role in clinical tests and public health. Nevertheless, each of them has distinct constrictions. As mentioned above, RT-PCR requires expensive equipment and a long time, while the antibody test has a lot to develop in sensitivity and specificity.

There's a common method that works as a supporting role in the diagnosis of SARS-CoV-2 is CT. It can divide into two categories: industrial CT and medical CT. They are both based on the same underlying physics principles but different in system and design because of the application types. Industrial CT scanners are usually used for quantitative dimensional analysis [6]. In contrast, medical CT scanners are optimized for qualitative viewing (image quality of human subjects) and specifically optimized for a low dose [7]. At the same time, dimensional accuracy is not crucial for medical diagnosis [6].

Moreover, other methods like historical epidemiology can be used as a reference. It indicates whether the patient is more likely to get the disease due to his/her ancestors, living conditions, daily diets, etc.

\section{The Pathology of SARS-CoV-2}

The receptor-binding domain and multi-base cleavage site on the SARS-CoV-2 spike protein are the distinguishing features that distinguish it from other related coronaviruses [8]. But these two characteristics are completely different from what humans can create with known coronaviruses. Therefore, SARS-CoV-2 cannot be man-made. It must be the result of natural selection.

As for the 1 hypothesis: SARS-CoV-2 has evolved the current pathogenic state in the non-human host. So far, the $\beta$-coronaviruses obtained from the bats mentioned above and pangolins have no multi-base cleavage sites.

But the collection of these animal viruses is not perfect, so it's irresponsible to conclude now. Suppose the animal host has a high population density and has an ACE2 encoding gene similar to a human homologous gene. In that case, the polybasic cleavage site can be formed in the animal [8].

As for the 2 hypotheses: SARS-CoV-2 jumps to the human before it evolves the current pathogenic state. After entering the human body, a precursor virus of SARS-CoV2 can undergo adaptive mutation into the current virus in the process of undetected human-to-human transmission [8]. Therefore, the insertion of the polybasic cleavage site can occur during human-to-human transmission. Still, current evidence lacks the stage of human-to-human transmission between the first transmission of the virus from animals to humans and the emergence of the final multi-base cleavage site. And there is a lack of serological studies that can determine the level of human exposure to SARS-CoV-2.

\section{Current situations of prevention and control}

As of April 13, 2021, compared with December 2020, both the number of confirmed cases and the number of deaths worldwide have decreased [9]. But the situation is not optimistic. The COVID-19 epidemic has still been repeated. Especially from February to April 2021, the number of confirmed cases and deaths worldwide has increased again [9]. Among them, the number of deaths in the United States and Europe is the highest globally, while China has maintained the number of confirmed cases at about 15 people per day since 2021 [9]. It is worth noting that countries are now starting to plant vaccines in large quantities, which will impact future data.

The above data shows that China, which was the first to have a large number of COVID-19 outbreaks, quickly contained the epidemic and has maintained an ultra-low number of new cases and deaths since April 2020. However, countries like America and Brazil have been unable to control the epidemic. This is due to the different policies of the two countries in dealing with the epidemic. Some countries, such as China, have adopted strict internal prevention and control, while others have adopted strict external prevention and control and more relaxed internal prevention and control. According to Dr. Qian's research, 
once there are people infected with the SARS-CoV-2 who have not been screened and isolated in a country, the trend of the epidemic in that country depends largely on the level of internal epidemic prevention and control in the country [10].

The government leads some countries to implement strict domestic epidemic control measures. And China is the best example. China used the gid government's method to effectively control the infection rate during the Spring Festival [11]. In the early stages of the pandemic, several provinces and cities in China initiated a first-level public health emergency response. The government closed down public entertainment venues, cordoned off communities, and strictly restricted the floating population. Moreover, the Chinese government advocated that people should self-isolate at home, suspend work and school, and check their temperature daily. And it is such strict internal prevention and control that effectively prevented the outbreak of the epidemic from spreading and enabled China to resume production in April 2020. Today, when most countries are still facing the threat of the new crown virus, China has gotten rid of the virus.

On the contrary, other countries have adopted strict external prevention and control policies and more relaxed internal prevention and control policies. The United States and Europe are good examples. In the early stage of the epidemic, the United States, Australia, Indonesia, and other countries implemented the control policy of "prohibiting the entry of Chinese citizens and/or residents of other countries who have been in China within 14 days" to strictly manage entry and exit [10]. Dr. Qian used a quantitative regression model to compare countries that emphasize internal prevention and control with those that place more emphasis on external prevention and control and found that countries that place more emphasis on external prevention and control have relatively more people infected with COVID-19 [10]. This means that the stringent immigration control policies adopted by them have not had the effect of preventing and controlling the cross-border spread of the epidemic for these countries. He explained in a subsequent report: "The t-test based on the Google search index initially shows that compared with countries that implement relatively loose foreign defense policies, countries that implement stringent foreign defense policies have a higher degree of attention to internal epidemics. The awareness of prevention is relatively lax, which also preliminarily explains the phenomenon that strict foreign defense policies in the early stage of the epidemic did not effectively prevent the epidemic from spreading across the country."[10].

What is certain is that under the threat of the COVID19, various countries have adopted certain countermeasures, including internal prevention and control and external prevention and control. However, Asian countries such as China are more inclined to implement an epidemic prevention policy based on strict internal prevention and control. In contrast, European countries such as the United States are more inclined to strict external prevention and control.

As for the emergence of this situation, there are many reasons, including politics, economy, and culture.

From a cultural perspective, in the traditional East
Asian national culture, the family is the basic unit of society, and it is the foundation of wider society [11]. Collectivism and patriotism are the sources of mass cohesion [11]. The fight against the epidemic in China fully reflects this cultural characteristic. The outbreak of the COVID-19 coincided with the Spring Festival, when the Chinese must return to their hometowns to reunite with their families. However, during the Spring Festival of 2020, many people took the initiative to abandon their travel plans and stay in isolation at home with arrangements made by the country. The interests of the country and the collective are more important in Chinese minds, and they have shown a united ethnic complex in this epidemic.

However, Western countries advocate freedom and democracy even more. Since the renaissance, Western countries have been fighting for freedom. After centuries of edification, the sense of freedom has become deeply rooted in people's hearts. Therefore, citizens of Western countries will pay more attention to their rights and do not like to be restrained. They generally believe that the state does not have any illegitimate rights to deprive them of liberty, even in emergencies. Therefore, even during the epidemic of COVID-19, they still adhere to their plans and exercise their due rights. For example, western citizens held many demonstrations to wear masks, which would hinder freedom [10].

There is no difference in culture between good and bad, but different cultures did affect the efficiency of response and control during this epidemic. Nations with high levels of cultural tightness, such as the United Chinese culture, may be more effective in responding to COVID-19 because they can adopt cooperative behaviors. According to Dr. Gelfand's "our evolutionary game-theoretic model", when a group is threatened, compared to loose cultures, cooperative norms develop faster in a tight environment, which leads to a higher survival rate [12].

\section{Antiviral treatment}

\subsection{Remdesivir}

Remdesivir (GS-5734) is a viral RNA- dependent RNA polymerase inhibitor, which can inhibit the activity of SARS-CoV-1 and the Middle East respiratory syndrome (MERS-CoV) [13-16]. Remdesivir works by interfering with a necessary enzyme the virus needs to make replication of RNA. This can prevent the viruses from multiplying as the genomes of coronaviruses are made up of ribonucleic acid (RNA). Also, remdesivir can cause side effects such as severe headache, nausea, fever, etc.

\subsection{Favipiravir}

According to existing clinical data, compare with remdisivir, favipiravir has a better therapeutic effect and fewer side effects [17]. The working mechanism of favipiravir is similar to remdesivir, as RNA polymerase inhibitors. Moreover, China's National Medical Products Administration has approved favipiravir as the first antiCOVID-19 drug in China because there are clinical trials 
that had demonstrated efficacy with minimal side effects in March [18].

\subsection{Lopinavir/Ritonavir and other HIV protease inhibitors}

Lopinavir-ritonavir combination is an inhibitor of protease of human immunodeficiency virus. Some clinical trials in the past have demonstrated that the lopinavir-ritonavir combination can be used to treat severe acute respiratory distress syndrome (SARS). Since the coronavirus has many similarities with the SARS virus [19], the potency of the lopinavir-ritonavir combination has been initiated to be tested by clinical trials. A randomized, controlled, open-label trial (ClinicalTrials.gov: ChiCTR2000029308) was initiated. 199 confirmed patients with COVID-19 participated in this trial. However, there did not show benefit from using lopinavir-ritonavir combination drugs [20].

\subsection{Ivermectin}

Ivermectin is a Food and Drug Administration (FDA)approved antiparasitic drug, treating several tropical diseases. It has been widely used and is generally well tolerated. It can dissociate the preformed IMP $\alpha / \beta 1$ heterodimer responsible for the nuclear transport of viral protein cargos [21]. It is a feasible therapeutic approach to the treatment of COVID-19 [22, 23] since nuclear transport is essential for the viral replication and inhibition of the host's antiviral response. An in vivo study has recently proven Ivermectin's capability to reduce viral RNA up to 5,000-fold after $48 \mathrm{~h}$ of infection with SARSCoV-2 [24]. Ivermectin efficacy is still needed to prove. The next step is figuring out the adequate dosing by trials. Ivermectin is not approved by the FDA for any treatment of viral infection so far.

\subsection{Chloroquine or hydroxychloroquine with or without Azithromycin}

Chloroquine has been used for more than 70 years and was first used to treat malaria therapy. The pharmacological effect of hydroxychloroquine is similar to that of chloroquine. The structure of the SARS-CoV-2 virus is similar to that of SARS CoV. Real-time RT-PCR has verified the similarity between the SARS CoV-2 gene sequence and the SARS-CoV gene sequence [25]. Since both SARS-CoV viruses and SARS-CoV-2 viruses infect the human body by combining with ACE2, hydroxychloroquine can inhibit the replication of viruses by interfering with this combination [26]. In recent vitro testing revealed its ability that chloroquine can reduce the viral cope number of SARS-CoV-2 effectively [27-34].

\section{Vaccine}

However, at present, there is no direct evidence to prove that a certain antiviral drug is effective in treating Covid19 , and the therapeutic effect of these new antiviral drugs needs further observation. Therefore, the development and use of vaccines have still been the key and hot spot to solve the Covid-19 epidemic. As of April 6, 2021, a total of 86 vaccines were in clinical development, and 186 vaccines were in the preclinical stage. The vaccines in the clinical stage include 28 Protein subunit vaccines, 12 viral vector vaccines (non-replicating), 10 DNA vaccines, 11 Inactivated vaccines, 12 RNA vaccines, 4 viral vector vaccines, 4 viruses like particle vaccines, $2 \mathrm{VVr}+$ Antigen Presenting Cell vaccines, 2 Live Attenuated Virus vaccines, and $1 \mathrm{VVnr}+$ Antigen Presenting Cell vaccine. Table1show the dose and schedule of these vaccines. 11 kinds only need one shot. A total of 55 species need to be vaccinated with two injections, among which 6 species need to be vaccinated with the second injection 14 days after the first injection, 21 species need to be vaccinated again after 21 days, and 28 species need to be vaccinated again after 28 days. There is only one kind that needs three injections. The time is 28 days and 56 days after the first injection. Others did not have specified injection doses and schedules. For the route of administration, there are 2 kinds of oral COVID-19 vaccines and 71 kinds of injection vaccines, including 3 kinds of subcutaneous injection, 3 kinds of intradermal injection, 65 kinds of intramuscular injection, and 7 kinds of intranasal injection, which are shown in Table2[35].

Table 1. Number of different platforms of candidates in clinical phase [35]

\begin{tabular}{|c|c|c|c|}
\hline Platform & & $\begin{array}{c}\text { Candidate } \\
\text { vaccines (no. } \\
\text { and } \%)\end{array}$ & \\
\hline PS & Protein subunit & 28 & $33 \%$ \\
\hline VVnr & $\begin{array}{c}\text { Viral Vector } \\
\text { (non-replicating) }\end{array}$ & 12 & $14 \%$ \\
\hline DNA & DNA & 10 & $12 \%$ \\
\hline IV & $\begin{array}{l}\text { Inactivated } \\
\text { Virus }\end{array}$ & 11 & $13 \%$ \\
\hline RNA & RNA & 12 & $14 \%$ \\
\hline $\mathbf{V V r}$ & $\begin{array}{l}\text { Viral Vector } \\
\text { (replicating) }\end{array}$ & 4 & $5 \%$ \\
\hline VLP & $\begin{array}{l}\text { Virus Like } \\
\text { Particle }\end{array}$ & 4 & $5 \%$ \\
\hline $\begin{array}{l}\mathbf{V V r}+ \\
\text { APC }\end{array}$ & $\begin{array}{l}\text { VVr + Antigen } \\
\text { Presenting Cell }\end{array}$ & 2 & $2 \%$ \\
\hline LAV & $\begin{array}{l}\text { Live Attenuated } \\
\text { Virus }\end{array}$ & 2 & $2 \%$ \\
\hline \multirow[t]{2}{*}{$\begin{array}{c}\text { VVnr + } \\
\text { APC }\end{array}$} & $\begin{array}{l}\text { VVnr + Antigen } \\
\text { Presenting Cell }\end{array}$ & 1 & $1 \%$ \\
\hline & & 86 & \\
\hline
\end{tabular}


Table 2. Number of doses, schedule, and route of administration of candidates in clinical phase [35]

\begin{tabular}{|c|c|c|c|}
\hline $\begin{array}{c}\text { Number of doses } \\
\text { \& schedule }\end{array}$ & & $\begin{array}{c}\text { Candidate } \\
\text { vaccines (no. } \\
\text { and \%) }\end{array}$ & \\
\hline 1 dose & & 11 & $13 \%$ \\
\hline Day 0 & & 11 & \\
\hline 2 doses & & 55 & $64 \%$ \\
\hline Day $0+14$ & & 6 & \\
\hline Day $0+21$ & & 21 & \\
\hline Day $0+28$ & & 28 & \\
\hline 3 doses & & 1 & $1 \%$ \\
\hline Day $0+28+56$ & & 1 & \\
\hline $\begin{array}{l}\text { TBD / No Data } \\
\text { (ND) }\end{array}$ & & 19 & $22 \%$ \\
\hline & & 86 & \\
\hline $\begin{array}{c}\text { Route of } \\
\text { administration }\end{array}$ & & & \\
\hline Oral & & 2 & $2 \%$ \\
\hline Injectable & & 71 & $83 \%$ \\
\hline $\mathrm{SC}$ & $\begin{array}{c}\text { Sub } \\
\text { cutaneous }\end{array}$ & 3 & $3 \%$ \\
\hline ID & Intra dermal & 3 & $3 \%$ \\
\hline IM & $\begin{array}{l}\text { Intra } \\
\text { muscular }\end{array}$ & 65 & $76 \%$ \\
\hline IN & Intra nasal & 7 & $8 \%$ \\
\hline $\begin{array}{l}\text { TBD / No Data } \\
\text { (ND) }\end{array}$ & & 13 & $15 \%$ \\
\hline
\end{tabular}

\subsection{Protein subunit vaccine}

Protein subunit vaccine is a vaccine made by extracting the special protein structure of bacteria and viruses through chemical decomposition or controlled protein hydrolysis, and screening out immunologically active fragments, also called a component vaccine. Nowadays, recombinant subunit vaccine is widely used. The target antigen gene of bacteria or virus is constructed on the expression vector by recombination, then transformed into the receptor cell, induced to express antigen protein, and purified to make the vaccine. The well-known hepatitis B vaccine, hepatitis $E$ vaccine, and cervical cancer vaccine all adopt this platform.

The protein subunit vaccine jointly developed by the Institute of Microbiology, Chinese Academy of Sciences, and Anhui ZhifeilongKema Biopharmaceutical Co., Ltd., was approved for emergency use in China on March 19th, 2021. The vaccine uses RBD dimer as its vaccine antigen. In addition to expressing one RBD monomer (amino acid residues 319-541), two copies of RBD coding gene fragments (amino acid residues 319-537) were cloned in series, resulting in the expression of $60 \mathrm{kDa}$ homodimer, which can increase the stability of vaccine antigen [36]. The recombinant subunit vaccine has been widely studied because of its high safety and high production costeffectiveness. However, because it only uses a part of the pathogen as a new antigen protein, it inevitably reduces the antigenicity of the recombinant protein to a certain extent, which can weaken the immune response of the body and make the immune effect worse. A certain amount of adjuvant should be added to maintain a lasting and effective immune response.

\subsection{Viral Vector vaccine (VVr/VVnr)}

A viral vector vaccine is a vaccine constructed by embedding pathogen antigen into other specific viral vectors or bacterial vectors (such as adenovirus, influenza virus, Salmonella, etc.), including replication type and non-replication type. According to different types of viral vectors, the immune life of viral vector vaccines is also different. At present, adenovirus is commonly used as a vector because of its ability to induce humoral immunity and cellular immunity. Meanwhile, its production cost is also lower [37].

Ad5 adenovirus vector vaccine $\mathrm{Ad} 5-\mathrm{nCoV}$, jointly developed by academician Chen Wei of the academy of military medical sciences and kangxinuo biology, was launched in China on February 25th, 2021. According to the third-phase clinical data, the overall protective effect of the vaccine on all symptoms after 28 days of single-shot vaccination is $65.28 \%$; After 14 days of single-shot vaccination, the protective effect was $68.83 \%$. The vaccine's protective efficacy against severe symptoms is $90.07 \%$ after 28 days of single injection vaccination and $95.47 \%$ after 14 days of single-shot vaccination, proving that the vaccine had good immunogenicity. Whereas, due to pre-existing immunity to $\mathrm{HAdV}-\mathrm{C} 5$, sometimes the antibody and $\mathrm{T}$ cell immune reaction of this vaccine will be reduced, which cannot give full play to the effect [38].

\subsection{Nucleic acid vaccine (DNA/RNA)}

The nucleic acid vaccine is a new vaccine development technology in recent years, including the DNA vaccine and mRNA vaccine. Its mechanism is to construct a DNA plasmid or mRNA fragment as an immunogen in vitro, subsequently introduce it into the human body by plasmid injection or making mRNA nanoparticles, and synthesize protein antigen in vivo to induce immune response[39]. It generally has the advantages of convenient construction and low production cost. But it is difficult to transport nucleic acid because of the harsh preservation conditions. Moreover, it is unstable and has the risk of integrating into the nuclear genome. There is no listed nucleic acid vaccine applied to the human body before, and the only AIDS 
vaccine, the Zika virus vaccine, is only in the clinical trial stage.

A widely used nucleic acid vaccine nowadays named BNT162b2 led by Pfizer Biotechnology Company of America was authorized for emergency use for the first time in Britain on December 2, 2020, and was conditionally approved for the first time in Switzerland on December 19, 2020. It was used for active immunization to prevent Covid-19 caused by novel coronavirus virus in people aged 16 and above. Most systemic events were mild to moderate severity. Severe systemic events occurred in $<2 \%$ of BNT $162 \mathrm{~b} 2$ recipients after each dose, except for fatigue and headache after the second dose (occurring in $4 \%$ and $2 \%$, respectively) [40].

However, according to the specific data of clinical application of Pfizer vaccine, there are still several reports of adverse reactions compared with vaccines of other platforms. The evaluation of its safety needs further evidence of clinical data.

\subsection{Inactivated vaccine}

The inactivated vaccine is a vaccine made by inactivating virus pathogen by heating or chemical agent, which makes it lose its virulence, but still retains its immunogenicity. Inactivated vaccines currently used include influenza, poliomyelitis, rabies, hepatitis A, intestinal EV71, and DTP vaccines. Because the pathogenic microorganism of the inactivated vaccine has been killed, it cannot grow and reproduce after entering the human body. Even vaccination of immunodeficiency patients will not cause infection or disease. However, it usually needs a large dose of injection and multiple vaccinations to enhance antibody titer. Inactivated vaccines mainly cause humoral immunity and rarely even do not cause cellular immunity. They are stable and easy to store.

PiCoVacc, developed by China Kexing Biopharmaceutical Company, has been put into widespread use. PiCoVacc vaccine is a multivalent vaccine preparation prepared by culturing virus in Vero cell line, collecting virus supernatant, inactivating and purifying virus with $\beta$-propiolactone, and adding alum adjuvant. It can be applied to virus strains prevalent in many regions. The mouse and rat experiments results showed that SARS-CoV-2 specific IgG with high titer was obtained in experimental animals inoculated with PiCoVacc. The neutralizing titer of serum could reach EC50 10e4 six weeks after injection, which was about 10 times higher than that of serum of rehabilitation patients in Covid-19. At the same time, the immune serum has a broad spectrum of reactions and can neutralize 10 different SARS-CoV-2 strains. In the rhesus monkey test results, the animals were vaccinated with $3 \mathrm{ug}$ and 6ug of PiCoVacc ACC, which simulated the vaccination situation of human beings. Seven days after injection, neutralizing antibodies with high titer (10e3) were produced in rhesus monkeys, which illustrated the high protective power of the vaccine. Previous clinical data presented that PiCoVacc rarely produced serious adverse reactions, which indicated that PicoVACC was safe in the meantime.

\subsection{Virus-Like Particle vaccine}

Virus-like particles (VLPs) are hollow particles containing one or more structural proteins of a certain virus, which have no viral nucleic acid and cannot replicate autonomously. They are the same or similar in morphology to real virus particles. Because of poor immunogenicity, VLP preparations usually need adjuvant to improve their efficiency. 3 VLP-based vaccines for human use have been officially approved to prevent hepatitis B virus, human papillomavirus, and hepatitis E virus.

The VLP vaccine developed in Canada has entered the Phase II/III clinical trial. In its phase I clinical trial, the researchers used two adjuvants to test the candidate vaccine. The mid-term analysis showed that the vaccine with VLP plus adjuvant was well tolerated; After two doses of adjuvant vaccine, the subjects produced obvious antibody and cellular immunity, and the neutralizing antibody in the lowest dose group with adjuvant was 10 times higher than that in the convalescent serum [39].

\subsection{Live Attenuated Virus vaccine}

Live attenuated vaccine means that after the pathogen is treated with formaldehyde, the toxic subunit structure changes, and the toxicity decreases, but the activity of the binding subunit remains unchanged, and its antigenicity remains. Inoculating it into the body can cause an immune reaction without causing diseases. Compared with inactivated vaccine, this kind of vaccine has stronger immunity and a longer action time. However, in some cases, the virulence reversal of live attenuated vaccine [41] will lead to tissue damage and infection of immunocompromised host, so its safety has certain hidden dangers. Vaccines listed include BCG, polio, measles, Japanese encephalitis, hepatitis A, etc.

Because of its safety limitation, only a few vaccines have entered the clinical stage. Up to April 2021, two LAV vaccines developed by Meissa Vaccines, Inc, the Indian Institute of Serology, and Griffith University have entered phase I clinical trials.

\subsection{Other treatment}

There are many other treatments for Covid-19, which are widely used in clinics together with antiviral drugs and vaccines, including general treatment, immunotherapy, glucocorticoid therapy, and traditional Chinese treatment.

\subsubsection{General treatment}

Covid-19 patients need to pay attention to bed rest, strengthen support treatment, ensure adequate energy intake; Pay attention to the balance of water and electrolyte, and keep the internal environment stable. Give effective oxygen therapy measures timely, including nasal catheter, mask oxygen supply, and nasal high-flow oxygen therapy. It can be treated with hydrogen and oxygen mixed inhalation gas $(\mathrm{H} 2 / \mathrm{O} 2: 66.6 \% / 33.3 \%)$ [42]. If necessary, critically ill patients, circulatory support, anticoagulant 
therapy, renal replacement therapy, and blood purification therapy should be adopted.

\subsubsection{Passive immunotherapy}

\subsubsection{Convalescent plasma}

It is suitable for severe and critical patients with rapid disease progression. In a study of 10 patients, 5 patients had significantly higher neutralizing antibody levels (1:640). In another study, neutralizing antibody levels in all five patients increased after blood transfusion (40-60 before blood transfusion and 80-320 on the 7th day). Other research also revealed that most patients who use $\mathrm{CP}$ therapy would get negative results of virus nucleic acid detection within 12 days [43, 44].

\subsubsection{Intravenous immunoglobulin}

IVIG is usually used for adjuvant treatment of critically ill patients. As a purified plasma product, protein is the main component, abundant in viral IgG. Continuous infusion can increase IgG levels in serum, effectively neutralize the antigen in patients' respiratory tract, and promote the recovery of diseases. IVIG can enhance the body's selfdefense, inhibit receptors on target cells, and prevent pathogens from binding and invading target cells. In addition, IVIG can also hinder the normal immune response of leukocytes and inhibit the production of inflammatory factors, thus reducing the inflammatory injury experienced by patients. A study has shown that IVIG can improve the indexes of patients with severe pneumonia in Covid-19 in a short time, improve patients' treatment efficiency, and have high effectiveness. [45].

Covid-19 may be related to immune imbalance and excessive inflammation, leading to or aggravating acute respiratory distress syndrome and multiple organ failure. A high level of interleukin-6 was positively correlated with severe Covid-19 symptoms. In addition, it has been found that the increased level of interleukin-6 is a predictor of the possibility of mechanical ventilation. Therefore, the anti-IL-6 receptor monoclonal antibody, Tocilizumab, is considered to be possibly efficient for the treatment of Covid-19 pneumonia [46].

Some moderately conclusive evidence indicates that Tocilizumab can reduce the risk of mechanical ventilation in hospitalized Covid-19 patients. Although RcT showed that Tocilizumab could not reduce short-term mortality, the low-certainty evidence from cohort studies revealed a link between Tocilizumab and lower mortality [47].

\subsubsection{Glucocorticoid therapy}

Glucocorticoids have been widely used in syndromes closely related to Covid-19, including SARS, Middle East Respiratory Syndrome, and influenza pneumonia. A retrospective study in China in 2006 showed that the rational use of glucocorticoids in severe SARS patients could reduce mortality and shorten the length of stay [48]. Another study on MERS revealed that corticosteroid treatment was not related to 90-day mortality but was related to the delay of MERS-CoV RNA clearance [49]. A meta-analysis found that glucocorticoid treatment is associated with high mortality and high infection rate in patients with influenza pneumonia, whether mild or severe[50]. These researches illustrate the positive and negative effects of hormones in viral pneumonia.

For Covid-19, the results of a study showed that the 28-day mortality rate of patients who used dexamethasone for 10 days in Covid-19 was lower than that of patients who randomly received invasive mechanical ventilation and non-invasive mechanical ventilation. Due to the lack of reliable evidence from large-scale randomized clinical trials, it has not been proved that dexamethasone has any benefit for patients who did not receive respiratory support when randomized. However, some evidence demonstrates that glucocorticoid dosage is different in terms of medical conditions and disease severity. The beneficial effect of glucocorticoid in severe viral respiratory infection may depend on the right dose, the right time, and among the right patients. Blind high doses may do more harm than good and cause greater harm to the human body. Therefore, although glucocorticoids have been recommended for severe cases in China, there are still great differences in practice worldwide. In many treatment guidelines, glucocorticoids are either prohibited or not recommended [51].

\subsubsection{Chinese medicine}

A summary study presented that the proportion of severe cases in patients treated with Chinese and Western medicine is lower than that of patients treated with Western medicine only. The fever duration and hospitalization time are also shorter. CM therapy is more likely to promote the absorption of lesions in CT scanning. In the meantime, the proportion of patients with improved clinical symptoms in the combination group was higher than in the simple western medicine group [43].

\section{Conclusion}

This article introduced the current observed feature and mechanism of SARS-CoV-2 and the current status of coronavirus disease, namely COVID-19. In addition to an overview of the COVID-19 pandemic, we also summarize the early diagnosis, the current prevention and control situation, treatment methods already in use, or potential treatment at the research stage, including antiviral treatments and vaccinations.

Moreover, we present several therapies which have been put into use in clinical treatment. Although there are currently no effective drugs for treating COVID-19, several existing therapies are helpful to treat symptoms associated with coronavirus infection.

This review article could be helpful for those who want to have a clear understanding of the structure and function of SARS-CoV-2 and the current status of the COVID-19 pandemic. Furthermore, the data and results presented in this paper can be used as reference information for those who plan to conduct COVID-19 related studies. 


\section{Reference}

1. Britannica, The Editors of Encyclopaedia. "Coronavirus". Encyclopedia Britannica, 23 Feb. 2021,

https://www.britannica.com/science/coronavirusvirus-group. Accessed 30 May 2021.

2. Li, Fang et al. "Structure of SARS coronavirus spike receptor-binding domain complexed with receptor." Science (New York, N.Y.) vol. 309, 5742 (2005): 1864-8. doi:10.1126/science. 1116480

3. Seyed Hosseini, Elahe et al. "The novel coronavirus Disease-2019 (COVID-19): Mechanism of action, detection and recent therapeutic strategies." Virology vol. 551 (2020): 1-9. doi:10.1016/j.virol.2020.08.011

4. "Coronavirus". Who.Int, 2021, https://www.who.int/healthtopics/coronavirus $\#$ tab $=$ tab_1

5. "The latest development of diagnostic testing technology in Covid-19". CAS, 2021, https://www.cas.org/zh-hans/blog/covid-19diagnostic-testing.

6. du Plessis, Anton et al. "Comparison Of Medical And Industrial X-Ray Computed Tomography For NonDestructive Testing". Case Studies In Nondestructive Testing And Evaluation, vol 6, 2016, pp. 17-25. Elsevier BV, doi:10.1016/j.csndt.2016.07.001.

7. Bartscher, M. et al. "Enhancement And Proof Of Accuracy Of Industrial Computed Tomography (CT) Measurements". CIRP Annals, vol 56, no. 1, 2007, pp. 495-498. Elsevier BV, doi:10.1016/j.cirp.2007.05.118.

8. Andersen, Kristian $\mathrm{G}$ et al. "The proximal origin of SARS-CoV-2.” Nature medicine vol. 26, 4 (2020): 450-452. doi:10.1038/s41591-020-0820-9

9. WHO Coronavirus Disease (COVID-19) Dashboard, 11 Sept. 2020, covid19.who.int.

10. Qian Xuesong, Ding Hai, and Zheng Dechang. "The COVID-19 epidemic, the early prevention and control measures of the international community and the evaluation of their effects-an empirical study based on the perspectives of external prevention and internal control." Financial Research 47.03 (2021): 4-18. doi: 10.16538 /j.cnki.jfe.20201115.403.

11. Wei, Yujun et al. "COVID-19 prevention and control in China: grid governance." Journal of public health (Oxford, England) vol. 43, 1 (2021): 76-81. doi:10.1093/pubmed/fdaa175

12. Gelfand, Michele J et al. "The relationship between cultural tightness-looseness and COVID-19 cases and deaths: a global analysis." The Lancet. Planetary health vol. $5, \quad 3 \quad$ (2021): e135-e144. doi:10.1016/S2542-5196(20)30301-6

13. Sheahan TP, Sims AC, Leist SR, et al. Comparative therapeutic efficacy of remdesivir and combination lopinavir, ritonavir, and interferon-beta against MERS-CoV. Nat Commun 2020; 11:222-222.

14. Agostini ML, Andres EL, Sims AC, et al. Coronavirus susceptibility to the antiviral remdesivir (GS-5734) is mediated by the viral polymerase and the proofreading exoribonuclease. mBio 2018;9(2):e00221-18-e00221-18.

15. Brown AJ, Won JJ, Graham RL, et al. Broad-spectrum antiviral remdesivir inhibits human endemic and zoonotic deltacoronaviruses with a highly divergent RNA dependent RNA polymerase. Antiviral Res 2019; 169:104541-104541.

16. Sheahan TP, Sims AC, Graham RL, et al. Broadspectrum antiviral GS-5734 inhibits both epidemic and zoonotic coronaviruses. Sci Transl Med 2017; 9:eaal3653-eaal3653.

17. Peng Q, Peng R, Yuan B, et al. Structural basis of SARS-CoV-2 polymerase inhibition by Favipiravir. Innovation (N Y), 2021. DOI: 10.1016/j.xinn.2021.100080

18. Yung-Fang Tu, Chian-Shiu Chien, et al. A Review of SARS-CoV-2 and the Ongoing Clinical Trials. International journal of molecular sciences, 2020; p.9.

19. An overview of therapeutic drugs of COVID-19 and its mechanism LIU Shu-lei, LIU Wei. No. 960th Hospital of PLA, Tai'an, Shandong 271000, China

20. Yung-Fang Tu, Chian-Shiu Chien, et al. A Review of SARS-CoV-2 and the Ongoing Clinical Trials. International journal of molecular sciences, 2020; p.9.

21. Wagstaffff, K.M.; Sivakumaran, H.; Heaton, S.M.; Harrich, D.; Jans, D.A. Ivermectin is a specific inhibitor of importin alpha/beta-mediated nuclear importable to inhibit replication of HIV-1 and dengue virus. Biochem. J. 2012, 443, 851-856. [CrossRef]

22. Yang, S.N.Y.; Atkinson, S.C.; Wang, C.; Lee, A.; Bogoyevitch, M.A.; Borg, N.A.; Jans, D.A. The broad-spectrum antiviral ivermectin targets the host nuclear transport importin alpha/betal heterodimer. Antivir. Res. 2020, 104760. [CrossRef]

23. Caly, L.; Wagstaffff, K.; Jans, D.A. Nuclear trafficking of proteins from RNA viruses: Potential target for antivirals? Antivir. Res. 2012, 95, 202-206. [CrossRef] [PubMed]

24. Caly, L.; Druce, J.D.; Catton, M.G.; Jans, D.A.; Wagstaffff, K.M. The FDA-approved drug Ivermectin inhibits the replication of SARS-CoV-2 in vitro. Antivir. Res. 2020, 104787. [CrossRef] [PubMed]

25. Corman V M, Landt O, Kaiser M, et al. Detection of 2019 novel coronavirus (SARS-CoV-2) by real-time RT PCR [J]. Euro Surveill, 2020, 25(3): 2000045.

26. HE Wei, CHANG Bingcheng. Research progress on antiviral pharmacology and clinical research of chloroquine and hydroxychloroquine. Drug Evaluation Research. 2020, 1674-6376 (2020) 061015-06: p2.

27. Lan, L.; Xu, D.; Ye, G.; Xia, C.; Wang, S.; Li, Y.; Xu, H. Positive RT-PCR Test Results in Patients Recovered From COVID-19. JAMA 2020. [CrossRef]

28. Chen, J.; Lau, Y.F.; Lamirande, E.W.; Paddock, C.D.; Bartlett, J.H.; Zaki, S.R.; Subbarao, K. Cellular Immune Responses to Severe Acute Respiratory Syndrome Coronavirus (SARS-CoV) Infection in 
Senescent BALB/c Mice: CD4+ $T$ Cells Are Important in Control of SARS-CoV Infection. J. Virol. 2009, 84, 1289-1301. [CrossRef] [PubMed]

29. Duan, K. et al. Effectiveness of convalescent plasma therapy in severe COVID-19 patients. Proc. Natl Acad. Sci. USA 117, 9490-9496 (2020).

30. Shen, C. et al. Treatment of 5 critically ill patients with COVID-19 with convalescent plasma. JAMA $323,1582-1589$ (2020).

31. CHEN Bishan, CHEN Jisheng. Study on the application of golimumab in novel coronavirus pneumonia [J]. Pharmacology Today, 2020, 30(4): 225-228.

32. Zhu Yungui, Deng Ziwei, Liu Lihua, et al. New coronary pneumonia treatment protocols for the treatment of drug information compilation of drug information for the treatment of New Coronary Pneumonia (First Edition) [J]. Zhongnan Pharmacology, 2020, 18(3): 345-358.

33. COVID-19 treatment protocol (trial version 7) $[\mathrm{J}]$. Chinese Medicine, 2020, 15(6): 801-805.

34. ZHAO Cheng-qi, JIN Zeng-hua, WANG Xue-fen. Advances in the study of novel drugs for the treatment of COVID-19. Military Medical Journal of Southeast China, 2021, 23(1), 1672-271X( 2021) 01-0058-06

35. Draft landscape and tracker of COVID-19 candidate vaccines (World Health Organization. 2021.)

36. Pollet, Jeroen et al. "Recombinant protein vaccines, a proven approach against coronavirus pandemics." Advanced drug delivery reviews vol. 170 (2021): 71-82. doi:10.1016/j.addr.2021.01.001

37. Li, Lifeng et al. "SARS-CoV-2 vaccine candidates in rapid development. " Human vaccines \& immunotherapeutics vol. 17, 3 (2021): 644-653. doi:10.1080/21645515.2020.1804777

38. Zhu, Feng-Cai et al. "Immunogenicity And Safety Of A Recombinant Adenovirus Type-5-Vectored COVID-19 Vaccine In Healthy Adults Aged 18 Years Or Older: A Randomised, Double-Blind, PlaceboControlled, Phase 2 Trial". The Lancet, vol 396, no. 10249, 2020, pp. 479-488. Elsevier BV, doi: 10.1016/s0140-6736(20)31605-6.

39. Zhu, Yao et al." Research Progress of Vaccine in novel coronavirus." Preventive medicine 33.02(2021):143148. doi:10.19485/j.cnki.issn2096-5087.2021.02.009.

40. Lamb, Yvette N. "BNT162b2 mRNA COVID-19 Vaccine: First Approval." Drugs vol. 81, 4 (2021): 495-501. doi:10.1007/s40265-021-01480-7

41. Weinberg, Geoffrey A. "Live, Attenuated Influenza Vaccine: Present and Future Roles." Journal of the Pediatric Infectious Diseases Society vol. 9, Supplement_1 (2020): doi:10.1093/jpids/piz100

42. "Novel coronavirus diagnosis and treatment plan (trial eighth edition)." Chinese Journal of Clinical Infectious Diseases 13.05(2020):321-328. doi:.
43. Wang, Meng et al. "Evaluation of current medical approaches for COVID-19: a systematic review and meta-analysis." BMJ supportive \& palliative care vol. 11, 1 (2021): 45-52. doi:10.1136/bmjspcare-2020002554

44. Duan, Kai et al. "Effectiveness of convalescent plasma therapy in severe COVID-19 patients." Proceedings of the National Academy of Sciences of the United States of America vol. 117, 17 (2020): 9490-9496. doi:10.1073/pnas.2004168117

45. Xie, Yun et al. "Effect of regular intravenous immunoglobulin therapy on prognosis of severe pneumonia in patients with COVID-19." The Journal of infection vol. 81, 2 (2020): 318-356. doi:10.1016/j.jinf.2020.03.044

46. Salama, Carlos et al. "Tocilizumab in Patients Hospitalized with Covid-19 Pneumonia." The New England journal of medicine vol. 384, 1 (2021): 2030. doi:10.1056/NEJMoa2030340

47. Tleyjeh, Imad $M$ et al. "Efficacy and safety of tocilizumab in COVID-19 patients: a living systematic review and meta-analysis. " Clinical microbiology and infection: the official publication of the European Society of Clinical Microbiology and Infectious Diseases vol. 27, 2 (2021): 215-227. doi:10.1016/j.cmi.2020.10.036

48. Chen, Rong-Chang et al. "Treatment of severe acute respiratory syndrome with glucosteroids: the Guangzhou experience." Chest vol. 129, 6 (2006): 1441-52. doi:10.1378/chest.129.6.1441

49. Arabi, Yaseen $\mathrm{M}$ et al. "Corticosteroid Therapy for Critically Ill Patients with Middle East Respiratory Syndrome." American journal of respiratory and critical care medicine vol. 197, 6 (2018): 757-767. doi:10.1164/rccm.201706-1172OC

50. Yang, J-W et al. "Corticosteroid administration for viral pneumonia: COVID-19 and beyond." Clinical microbiology and infection: the official publication of the European Society of Clinical Microbiology and Infectious Diseases vol. 26, 9 (2020): 1171-1177. doi:10.1016/j.cmi.2020.06.020

51. RECOVERY Collaborative Group et al. "Dexamethasone in Hospitalized Patients with Covid19." The New England journal of medicine vol. 384, 8 (2021): 693-704. doi:10.1056/NEJMoa2021436 